\title{
Contribuição ao conhecimento da herpetofauna do nordeste do estado de Minas Gerais, Brasil
}

\author{
Renato Neves Feio $^{1}$ e Ulisses Caramaschi ${ }^{2,3}$ \\ 1 Museu de Zoologia João Moojen Oliveira, Departamento de Biologia Animal, Universidade Federal de Viçosa, \\ 36571-000 Viçosa, MG, Brasil. E-mail: rfeio@ufv.br. \\ 2 Museu Nacional/UFRJ, Quinta da Boa Vista, São Cristóvão, 20940-040 Rio de Janeiro, RJ, Brasil. E-mail: \\ ulisses@acd.ufrj.br. \\ 3 Bolsista do Conselho Nacional de Desenvolvimento Científico e Tecnológico (CNPq).
}

Keywords: Amphibians, reptiles, geographical distribution, Atlantic Rain Forest, Minas Gerais, Brazil.

Palavras-chave: Anfíbios, répteis, distribuição geográfica, Mata Atlântica, Minas Gerais, Brasil.

A Mata Atlântica consiste em um dos biomas mundiais que apresentam alta diversidade biológica e elevado índice de espécies endêmicas. Aliado à sua acelerada destruição, restando atualmente pouco mais de $5 \%$ de sua extensão original, foi declarada como uma das cinco primeiras colocadas nas regiões classificadas como "hotspots", ou seja, regiões biologicamente mais ricas e ameaçadas de todo o planeta (Myers et al. 2000).

Em Minas Gerais, fragmentos significativos ainda bem-preservados e legalmente protegidos de Mata Atlântica estão limitados a unidades de conservação nas regiões sul e sudeste do estado, com destaque para os Parques Nacionais do Itatiaia e do Caparaó (IBAMA), além dos Parques Estaduais do Rio Doce, Brigadeiro e Ibitipoca (IEF), sendo preliminares os estudos ambientais de diagnóstico sobre áreas significativas na região nordeste do estado (Camargos 2001).

Em relação à fauna, estudos realizados no século XIX pelos naturalistas Von Martius e Von Spix, entre 1817-1820, A. Saint-Hilaire, entre

Recebido em 25 de novembro de 2002

Aceito em 28 de dezembro de 2002
1816-1817, e expedições científicas, como a Thayer Expedition, entre 1865-1866, incluíram passagens pela região nordeste de Minas Gerais (Agassiz e Agassiz 1975, Saint-Hilaire 1975, Spix e Martius 1981). Estudos mais recentes (Carnevalli 1980, Mattos e Sick 1985, Rylands et al. 1988, Oliver e Santos 1991, Feio e Cotta 1992, Feio e Caramaschi 1995, Ribon et al. 2001, Feio et al. no prelo) caracterizaram alguns grupos de vertebrados no vale do rio Jequitinhonha, indicando áreas e regiões de interesse para preservação.

Durante viagens de reconhecimento a cinco fragmentos classificados como "floresta submontana ombrófila densa e estacional semidecidual", inseridos no bioma da Mata Atlântica (IBGE 1977, Veloso et al. 1991), tivemos a oportunidade de registrar dados relevantes sobre a herpetofauna regional, estimulando-nos a apresentar notas zoogeográficas que contribuam para o conhecimento da história evolutiva regional. As áreas amostradas são brevemente caracterizadas a seguir (Figura 1):

1) "Fazenda Alto Cariri” ( $\left.16^{\circ} 18^{\prime} \mathrm{S}, 39^{\circ} 59^{\prime} \mathrm{W}\right)$, no município de Salto da Divisa (MG), próximo aos limites do município de Itagimirim 
(BA). Essa fazenda, com 517 ha, encontra-se em região montanhosa, com altitudes variando entre 700 e $900 \mathrm{~m}$. Encontra-se contígua a outros fragmentos significativos de Mata Atlântica, formando um complexo vegetacional com aproximadamente 20.000 ha, podendo ser considerado um dos maiores remanescentes florestais nativos ainda bem-preservados do nordeste de Minas Gerais e sul da Bahia. Todo essa região abrange várias nascentes, constituindo-se em divisor das bacias dos rios Jequitinhonha, em Minas Gerais, e Buranhém, na Bahia, este com foz independente no Oceano Atlântico.

2) “Fazenda Limoeiro" (1603'S, 4051'W), localizada às margens do rio São Francisco, afluente da margem esquerda do rio Jequitinhonha, no município de Almenara (MG). A fazenda possui um grande fragmento de vegetação de Mata Atlântica, também contígua a outros remanescentes, perfazendo uma área total de aproximadamente 5.000 ha, com altitudes médias por volta de $800 \mathrm{~m}$. Possui também ambientes alterados por ação antrópica, como represas e brejos em pastos.

3) "Mata Escura" (16²0’ S, 4000’W), localizada na margem esquerda do rio Jequitinhonha, próximo à cidade de Jequitinhonha (MG). Consiste em grande extensão de mata em encosta, margeando toda a extensão do córrego Mata Escura. Essa área apresenta conexão com matas bem-preservadas do vale do córrego Labirinto, área de preservação da Companhia de Abastecimento e Saneamento de Minas Gerais (COPASA), formando um bloco contínuo de mata com cerca de 10.000 ha. Apresenta também, nos altos das chapadas, áreas abertas com solo arenoso, com grande incidência de bromeliáceas. Na única viagem realizada a essa região, somente esse ambiente de chapadas foi preliminarmente amostrado.

4) “Fazenda Ramaiana” (16² 42 'S, 4049’ W), localizada na divisa dos municípios de Joaíma e Felizburgo (MG), próximo a nascentes e

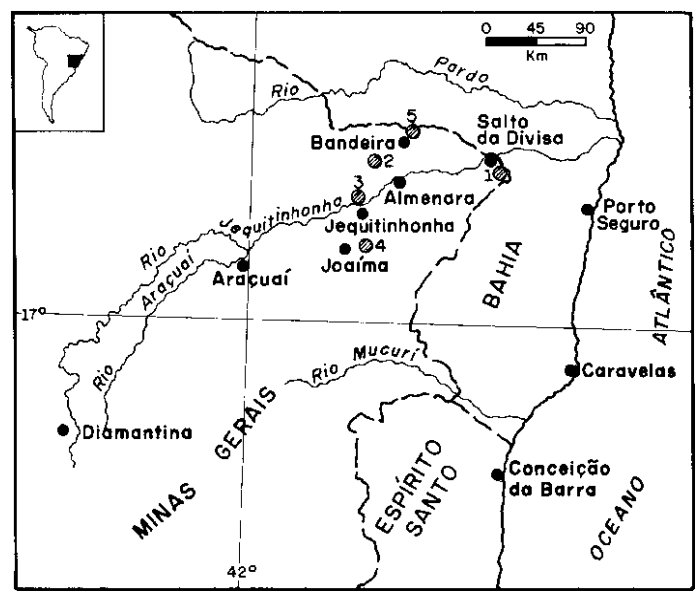

Figura 1 - Localização geográfica dos fragmentos de Mata Atlântica estudados no nordeste do estado de Minas Gerais, sudeste do Brasil: 1. Fazenda Alto Cariri; 2. Fazenda Limoeiro; 3. Mata Escura; 4. Fazenda Ramaiana; 5. Fazenda Mombuca.

córregos que fluem para a margem direita do rio Jequitinhonha. Apresenta-se como um fragmento linear de mata com aproximadamente 600 ha que margeia um córrego permanente, além de brejos e açudes artificiais nas proximidades da sede da fazenda.

5) "Fazenda Mombuca" ( $\left.15^{\circ} 47^{\prime} \mathrm{S}, 40^{\circ} 32^{\prime} \mathrm{W}\right)$, localizada próximo às nascentes do ribeirão do Norte, afluente da margem esquerda do rio Jequitinhonha, na divisa dos municípios de Bandeira (MG) e Macarani (BA). Apesar do fragmento de mata não ter sido efetivamente amostrado, dados obtidos com moradores locais foram relevantes, e uma das informações obtidas sobre a herpetofauna regional foi inserida nos resultados deste estudo.

As espécies foram diagnosticadas em observações (coleta e zoofonia) realizadas ao longo de trilhas regionais, concentrando-se em ambientes favoráveis à ocupação por anfíbios e répteis. As excursões ocorreram nas seguintes datas: 25-28 de setembro de 2000 (Fazenda Alto Cariri), 20 de fevereiro de 2001 (Mata Escura), 


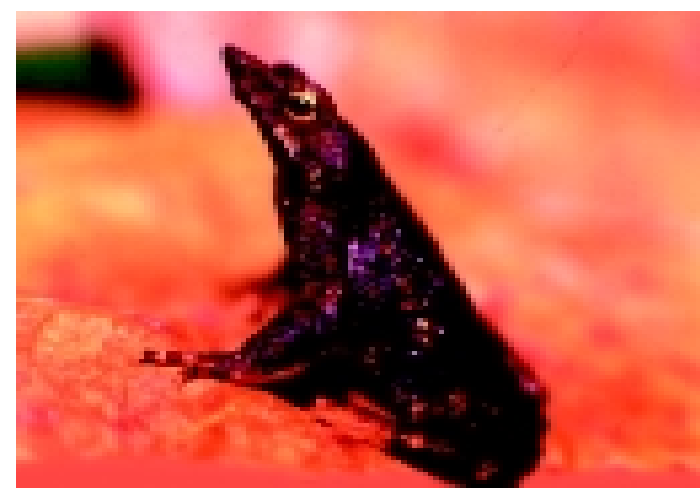

Figura 2 - Rhamphophryne proboscidea (Bufonidae).

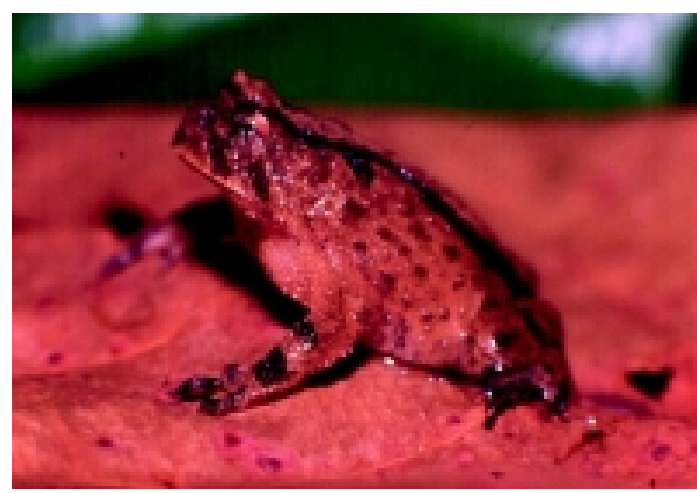

Figura 4 - Proceratophrys schirchi (Leptodactylidae).

21-22 de fevereiro de 2001 (Fazenda Limoeiro), 25-26 de abril de 2001 (Fazenda Ramaiana) e 18 de junho de 2002 (Fazenda Mombuca).

O material-testemunho encontra-se depositado no Museu de Zoologia "João Moojen de Oliveira", Departamento de Biologia Animal, Universidade Federal de Viçosa (MZUFV), e no Museu Nacional, Rio de Janeiro (MNRJ).

Foram registradas 30 espécies de anfíbios pertencentes às famílias Bufonidae (4), Hylidae (16), Leptodactylidae (9) e Pseudidae (1), além de 11 espécies de répteis, sendo cinco serpentes das famílias Colubridae (2) e Viperidae (3) e seis lagartos das famílias Gekkonidae (2), Polychrotidae (1), Teiidae (2) e Tropiduridae (1) (Tabela 1).

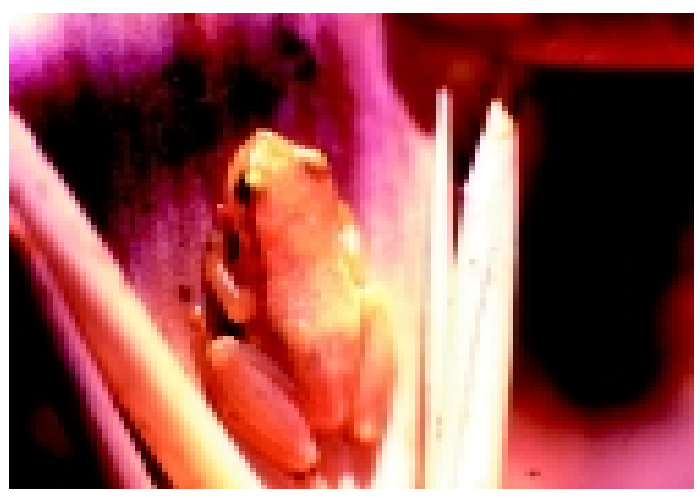

Figura 3 - Phyllodytes luteolus (Hylidae).

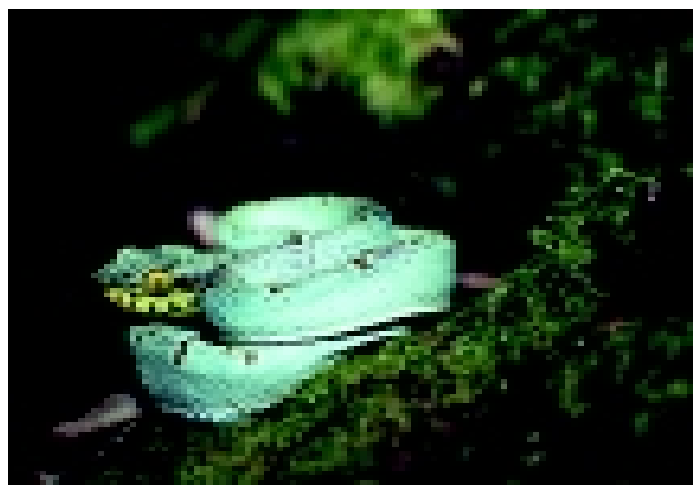

Figura 5 - Bothrops bilineatus (Viperidae).

Dentre os resultados encontrados, ainda que algumas espécies necessitem de material adicional para sua completa identificação, destacamse registros que representam novidades zoogeográficas e conservacionistas relevantes, merecendo comentários especiais.

Rhamphophryne proboscidea (Figura 2) é um pequeno bufonídeo que apresenta típico focinho proeminente, conhecido apenas da região metropolitana de Salvador (BA) e desaparecido desde 1930 (Izecksohn 1976). O registro desta espécie na Fazenda Limoeiro, em Almenara (MG), amplia significativamente sua distribuição geográfica, incorporando-a à anurofauna mineira. A espécie sobrevive em ambientes de interior de matas preservadas na 
região do baixo Jequitinhonha, indicando ainda sua ocorrência potencial em toda a faixa compreendida entre Salvador e essa região, o que representaria a maior distribuição de uma espécie desse gênero (Trueb 1971, Feio et al. no prelo).

Segundo Bokermann (1966), Peixoto e Cruz (1988) e Caramaschi et al. (1992), Phyllodytes compreende espécies que apresentam odontóides na mandíbula, caráter único entre os hilídeos, e que se reproduzem em broméliáceas, local de desenvolvimento dos girinos. No Brasil, Phyllodytes luteolus é conhecida apenas para o norte do estado do Espírito Santo e sul da Bahia. O registro de P. luteolus (Figura 3) na Mata Escura, na região do Jequitinhonha, consiste no primeiro registro de uma espécie do gênero para o estado de Minas Gerais, representando ainda o ponto mais continental na distribuição do gênero no Brasil.

Proceratophrys schirchi (Figura 4) é um pequeno leptodactilídeo de mata, conhecido dos estados do Espírito Santo e Rio de Janeiro (Caramaschi e Velosa 1997). O encontro dessa espécie nessa região consiste no primeiro registro para o estado de Minas Gerais, bem como representa um dos pontos mais setentrionais de distribuição da espécie.

O gênero Phasmahyla, segundo Cruz (1990) e Caramaschi e Cruz (2002), é definido, além de outros caracteres, pelo girino com boca em posição ântero-dorsal e expandida em forma de funil, encontrado em remansos de riachos e córregos de montanha florestada, na região sudeste do Brasil. O encontro de girinos de Phasmahyla sp. na Fazenda Alto Cariri, em Salto da Divisa (MG), representa ampliação significativa na distribuição do gênero, consistindo também no registro mais setentrional até o momento conhecido para Minas Gerais.

Dentre os répteis, apesar do pequeno número de espécies registradas, merece destaque a jararaca-verde ou jararaca-pingo-de-ouro, Bothrops bilineatus (Figura 5), que representa o primeiro registro dessa espécie em Minas Gerais. Esse dado também reforça a possibili- dade da sua presença em matas ainda mais interiores no vale do rio Jequitinhonha. Segundo Campbell e Lamar (1989), B. bilineatus é uma serpente peçonhenta arborícola que ocorre na Amazônia e na Mata Atlântica, nos estados do Rio de Janeiro, Espírito Santo e Bahia, podendo atingir $1 \mathrm{~m}$ de comprimento. Rocha et al. (2000) afirmaram que essa serpente possui baixa densidade populacional ao longo de sua área de distribuição no estado do Rio de Janeiro, bem como forte dependência por matas preservadas de encosta. Além disto, foi considerada como "provavelmente extinta" na Lista Oficial da Fauna Ameaçada de Extinção do Estado do Rio de Janeiro. O registro dessa espécie em Minas Gerais referenda sua inclusão na lista de animais ameaçados de extinção nesse estado.

Destaca-se também o registro de uma pele de surucuru-pico-de-jaca, Lachesis muta, procedente da Fazenda Mombuca, no município de Bandeira (MG), observada com um morador local. Essa espécie encontra-se oficialmente ameaçada de extinção em Minas Gerais e no Brasil (Bernardes et al. 1990, Cotta 1998), já tendo sido também registrada em outras regiões próximas, na Bahia (Franco et al. 1998).

Esses registros ampliam significativamente a distribuição geográfica de algumas espécies tidas como exclusivas de regiões litorâneas da Mata Atlântica, caracterizando o vale do rio Jequitinhonha como um dispersor de elementos da herpetofauna litorânea para o interior do Brasil, assim como já observado ao longo do rio Doce, no leste do estado de Minas Gerais (Feio et al. 1998, 1999). Além disso, reforçam a possibilidade da presença de outros representantes da herpetofauna típicos de regiões baixas da Mata Atlântica litorânea em outros fragmentos de mata localizados no vale do rio Jequitinhonha, como Hyalinobatrachium (Centrolenidae) e Crossodactylus (Leptodactylidae), já registradas em fragmentos mais interiores ao longo do rio Jequitinhonha por Feio e Caramaschi (1995). Acreditamos que novos esforços de observações e coletas na região proporcionarão dados extremamente recompensadores sobre a 
Tabela 1 - Espécies de anfíbios e répteis registradas no nordeste do estado de Minas Gerais, com a indicação da localidade, ambiente e forma de registro.

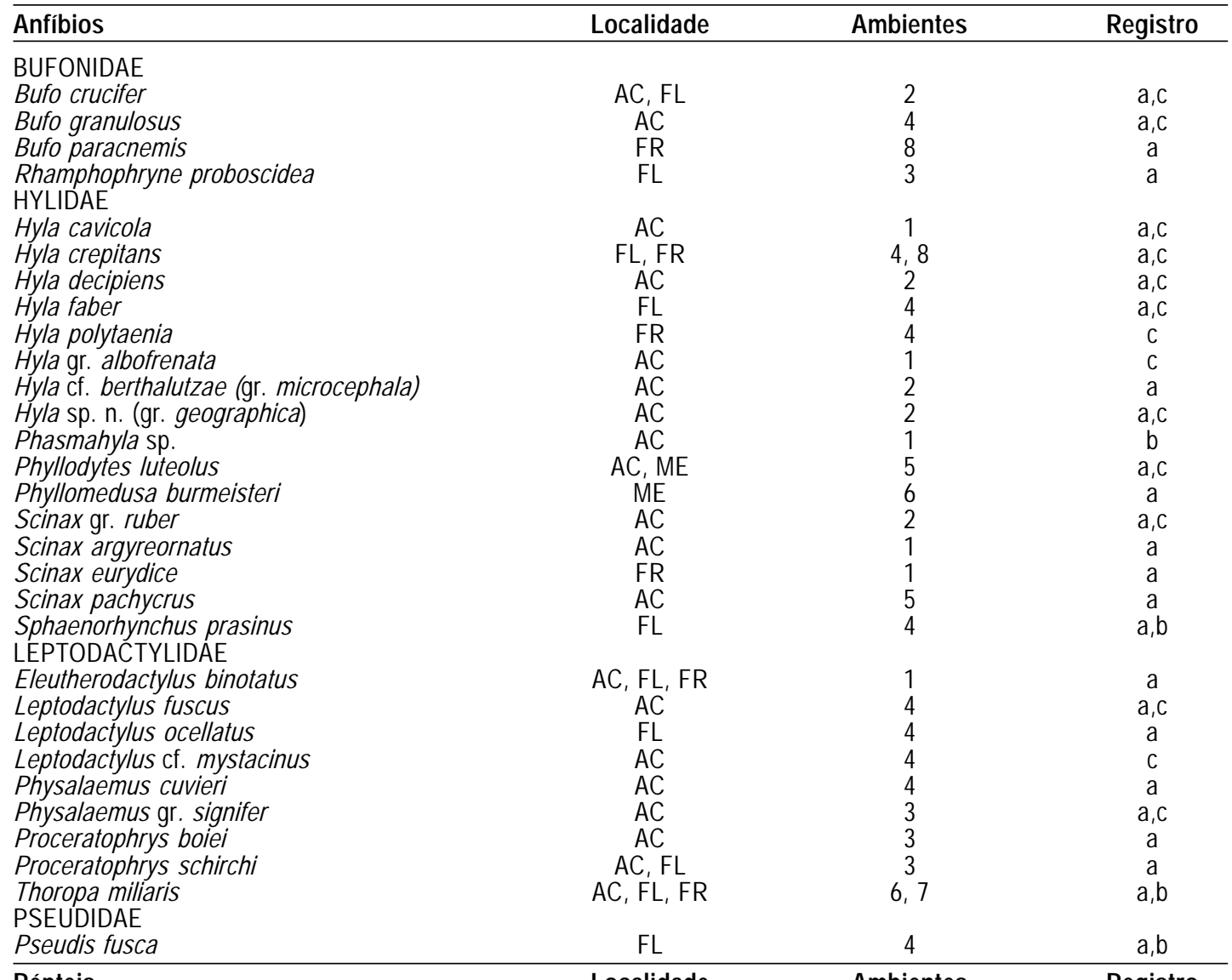

\begin{tabular}{|c|c|c|c|}
\hline Répteis & Localidade & Ambientes & Regis \\
\hline \multicolumn{4}{|l|}{ VIPERIDAE } \\
\hline $\begin{array}{l}\text { Bothrops bilineatus } \\
\text { Bothrops jararaca }\end{array}$ & $\begin{array}{l}A C \\
F R\end{array}$ & 6 & \\
\hline $\begin{array}{l}\text { Bothrops jararaca } \\
\text { Lachesis muta }\end{array}$ & $F M$ & & $\frac{a}{d}$ \\
\hline $\begin{array}{l}\text { COLUBRIDAE } \\
\text { Liophis miliaris }\end{array}$ & $\mathrm{FL}$ & 4 & a \\
\hline $\begin{array}{l}\text { Oxybelis aeneus } \\
\text { TEIIDAE }\end{array}$ & $A C$ & 6 & a \\
\hline Ameiva ameiva & $\mathrm{FL}$ & 4 & a \\
\hline $\begin{array}{l}\text { Cnemidophorus ocellifer } \\
\text { GEKKONIDAE }\end{array}$ & ME & 4,6 & a \\
\hline Gymnodactylus geckoides & $A C$ & 6 & a \\
\hline $\begin{array}{l}\text { Phyllopezus pollicaris } \\
\text { POLYCHROTIDAE }\end{array}$ & $\mathrm{FL}$ & 8 & a \\
\hline $\begin{array}{l}\text { Enyalius catenatus } \\
T R O P I D U R D A F\end{array}$ & $F R$ & 1 & a \\
\hline Tropidurus torquatus & $A C, F L, M E$ & 4 & $a$ \\
\hline
\end{tabular}

Localidades: AC - Fazenda Alto Cariri; FL - Fazenda Limoeiro; ME - Mata Escura; FR - Fazenda Ramaiana; FM - Fazenda Mombuca. Ambientes: (1) riachos de pequeno porte em interior de mata; (2) remansos de riacho de médio porte em borda de mata; (3) ambientes alagados em interior de mata; (4) ambientes alagados e/ou alterados em área aberta; (5) bromeliáceas epífitas e/ou terrestres em borda de mata; (6) estradas e acessos na mata; (7) afloramento rochoso em borda de mata; (8) habitações humanas. Forma de registro: (a) coleta de adultos; (b) coleta de girinos; (c) zoofonia e/ou visualização; (d) exemplar com morador local. 
composição da herpetofauna regional.

Com base nessas informações, constatou-se que a região do baixo rio Jequitinhonha apresenta remanescentes significativos e preservados de Mata Atlântica, com vários segmentos da herpetofauna típicos desse bioma, alguns bastante raros e com registros únicos no estado de Minas Gerais. A fauna regional deve ser urgentemente investigada em maior detalhamento para subsidiar prioridades na implantação de unidades de conservação.

\section{Agradecimentos}

Aos biólogos Fabiano R. Melo, Priscila M. Andrade, Denize F. Nogueira e Rômulo Ribon, pelo companheirismo durante os trabalhos de campo e/ou auxílio na caracterização das áreas de estudo; ao Instituto Estadual de Florestas de Minas Gerais, por meio do escritório regional de Teófilo Otoni (Paulo R. de Lauro) e escritório local em Jequitinhonha (Giovane A. Moura), pelo apoio local e deslocamento da equipe; aos Profs. Carlos Alberto G. Cruz, José P. Pombal Jr. e Eugenio Izecksohn, pelo auxílio nas identificações das espécies; ao Srs. Evídeo Aurich, Valdívio L. dos Santos e Leonor J. Botelho, pela permissão para trabalhar respectivamente nas Fazendas Alto Cariri, Limoeiro e Ramaiana, e também aos vários moradores/anfitriões locais pela amizade e logística nas atividades realizadas no campo.

\section{Referências Bibliográficas}

Agassiz, L. e E. C. Agassiz. 1975. Viagem ao Brasil: 18651866. São Paulo. Edusp/ Itatiaia. 323 pp.

Bernardes, A. T., A. B. M. Machado e A. B. Rylands. 1990. Fauna Brasileira Ameaçada de Extinção. Belo Horizonte. Fundação Biodiversitas. 65 pp.

Bokermann, W. C. A. 1966. O gênero Phyllodytes Wagler, 1830 (Anura, Hylidae). Anais da Academia Brasileira de Ciências. 38: 335-344.

Camargos, R. M. F. 2001. Unidades de Conservação em Minas Gerais: Levantamento e Discussão. Publicações Avulsas da Fundação Biodiversitas 2: 1-62.
Campbell, J. A. e W. W. Lamar. 1989. The Venomous Reptiles of Latin America. Cornell University Press. $425 \mathrm{pp}$.

Caramaschi, U. e C. A. G. Cruz. 2002. Phyllomedusa: posição taxonômica, hábitos e biologia (Amphibia, Anura, Hylidae). Phyllomedusa 1: 5-10.

Caramaschi, U. e A. Velosa. 1997. Stombus precrenulatus Miranda-Ribeiro, 1937, a junior synonym of Proceratophrys schirchi (Miranda-Ribeiro, 1937) (Anura: Leptodactylidae). Copeia 1977: 629-631.

Caramaschi, U., H. R. Silva e M. C. Britto-Pereira. 1992. A new species of Phyllodytes (Anura, Hylidae) from Southern Bahia, Brazil. Copeia 1992: 187-191.

Carnevalli, N. E. D. 1980. Primeiros Estudos sobre a Ornitofauna do Vale do Jequitinhonha em Minas Gerais. Relatório Final CETEC/UFMG. 86 pp.

Cotta, G. A. 1998. Lachesis muta rhombeata. Pp. 433435 in A. B. M. Machado, G. A. B. Fonseca, R. B. Machado, L. M. S. Aguiar e L. V. Lins (eds.), Livro Vermelho das Espécies Ameaçadas de Extinção da Fauna de Minas Gerais. Belo Horizonte. Fundação Biodiversitas.

Cruz, C. A. G. 1990. Sobre as relações intergenéricas de Phyllomedusinae da Floresta Atlântica (Amphibia, Anura, Hylidae). Revista Brasileira de Biologia 50: 709-726.

Feio, R. N. e U. Caramaschi. 1995. Aspectos zoogeográficos do anfíbios do alto/médio rio Jequitinhonha, Minas Gerais. Revista Ceres 42: 53-61.

Feio, R. N. e G. A. Cotta. 1992. Serpentes do alto/médio curso do rio Jequitinhonha, Minas Gerais. XII Congresso Latino-Americano de Zoologia, Belém. p. 187.

Feio, R. N., B. V. S. Pimenta e D. L. Silvano. Rediscovery and biology of Rhamphophryne proboscidea (Boulenger, 1882) (Anura, Bufonidae). AmphibiaReptilia. (no prelo).

Feio, R. N., P. S. Santos e U. Caramaschi. 1999. New records of amphibians from Parque Estadual do Rio Doce, State of Minas Gerais, Brazil. Herpetological Review 30: 56-57.

Feio, R. N., H. Wiederhecker, U. M. L. Braga e P. S. Santos. 1998. Anfíbios do Parque Estadual do Rio Doce. Viçosa. Universidade Federal de Viçosa/Instituto Estadual de Florestas. Imprensa Universitária. 32 pp.

Franco, F. L., G. O. S. Sugliano, M. Porto e O. A. V. Marques. 1988. Répteis na Estação Veracruz (Porto Seguro, Bahia). Veracel Celulose. 41 pp.

IBGE. 1977. Carta de Salto da Divisa. MI2275, Folha SE24.V.B-II. Escala 1:100.000. Belo Horizonte.

Izecksohn, E. 1976. O status sistemático de Phryniscus proboscideus Boulenger (Amphibia, Anura, Bufonidae). Revista Brasileira de Biologia 36: 341-345. 
Mattos, G. T. e H. Sick. 1985. Sobre a distribuição de duas espécies crípticas: Embernagra longicauda Strickland, 1844 e Embernargra platensis (Gmelin, 1789). Emberezidae, Aves. Revista Brasileira de Biologia 45: 201-206.

Myers, N., R. A. Mittermeier, C. G. Mittermeier, G. A. B. Fonseca e J. Kent. 2000. Biodiversity hotspots for conservation priorities. Nature 403: 853-858.

Oliver, W. L. R. e I. B. Santos. 1991. Threatened endemic mammals of the Atlantic Forest region of south-east Brazil. The Jersey Wildlife Preservation Trust, Special Scientific Report 4: 1-125.

Peixoto, O. L. e C. A. G. Cruz. 1988. Descrição de duas espécies novas do gênero Phyllodytes Wagler (Amphibia, Anura, Hylidae). Revista Brasileira de Biologia 48: 265-272.

Ribon, R., B. M. Whitney e J. F. Pacheco. 2001. Discovery of Bahia spinetail Synallaxis cinerea in north-east Minas Gerais, Brazil, with additional records of some rare and threatened montane Atlantic Forest birds. Cotinga 17: 52-56.

Rocha, C. F. D., M. V. Sluys, G. Puorto, R. Fernandes, J. D. Barros-Filho, R. R. S. F. Neo e A. Melgarejo. 2000.
Répteis. Pp. 79-87 in H. G. Bergallo, C. F. D. Rocha, M. A. S. Alves e M. Van Sluys (eds.), A Fauna Ameaçada de Extinção do Estado do Rio de Janeiro. Rio de Janeiro. EdUERJ, 166 pp.

Rylands, A. B., W. R. Spironelo, V. L. Tornisielo, R. L. Sá, M. C. M. Kierulff e I. B. Santos. 1988. Primates of the rio Jequitinhonha valley, Minas Gerais, Brazil. Primates Conservation 9: 100-109.

Saint-Hilaire, A. 1975. Viagem pelas Províncias do Rio de Janeiro e Minas Gerais. 4a edição. São Paulo. Edusp/ Itatiaia. $378 \mathrm{pp}$.

Spix, J. B. e K. F. P. Martius. 1981. Viagem pelo Brasil 1817-1820. 4a edição. São Paulo. Edusp/Itatiaia. 301 pp.

Trueb, L. 1971. Phylogenetic relationships of certain neotropical toads with the description of a new genus (Anura: Bufonidae). Contributions in Science Los Angeles Museum 6: 1-40.

Veloso, H. P., A. L. R. Rangel-Filho e J. C. A. Lima. 1991. Classificação da Vegetação Brasileira, Adaptada a um Sistema Universal. Rio de Janeiro. IBGE. Departamento de Recursos Naturais e Estudos Ambientais. $123 \mathrm{pp}$. 